\title{
Estrutura tecnológica e mudanças climáticas no Brasil: um estudo exploratório a partir de estatísticas de patentes
}

\author{
Paulo Henrique Assis Feitosa*
}

\section{RESUMO}

Este artigo analisa a evolução da estrutura tecnológica brasileira em áreas com potencial de mitigação de mudanças climáticas no período pós-Kyoto. A partir da constituição de uma base de dados original com patentes relacionadas às mudanças climáticas, propõe-se interpretar as transformações ocorridas na estrutura da especialização tecnológica do Brasil, bem como a evolução do seu desempenho em comparação a países selecionados. Os resultados da estimação de vantagens tecnológicas relativas mostram que o Brasil não acompanhou a dinâmica internacional e reduziu seu número absoluto de áreas com especialização tecnológica. A aplicação do método de decomposição estrutural revelou que o aumento da sua participação internacional é explicado, em sua maior parte, pelo efeito technology share, ou seja, a parte do crescimento que se deve ao dinamismo da sua atividade patenteadora. Nesse sentido, o país foi capaz de abandonar áreas estagnadas e os resultados seriam ainda mais favoráveis se contasse com uma melhor estrutura de especialização no período inicial.

PalaVras-ChaVE | Estrutura Tecnológica; Inovações Ambientais; Mudanças Climáticas; Especialização Tecnológica

CÓdigos-JEL | O33; O34; Q54; Q55

* Universidade Estadual de Campinas (Unicamp), Campinas (SP), Brasil. E-mail: paulohenriquefeitosa@gmail.com 


\title{
Technological structure and climate change in Brazil: an exploratory study from patent statistics
}

\begin{abstract}
This paper analyzes the evolution of the Brazilian technological structure in areas with potential to mitigate climate change in the post-Kyoto period. From the creation of an original database with patents related to climate change, the paper proposes an interpretation of the changes occurred in the technological specialization of the country and the evolution of its performance compared to selected countries. The Technological Revealed Comparative Advantage estimation showed that the country did not follow the international dynamics and reduced its number of areas of technological specializations. The structural decomposition analysis revealed that the increase in the Brazilian share of the world's patenting activity is explained mostly by the technology share effect, that is, by the part of the growth which is due to the dynamism of its patenting activity. The country abandoned stagnant areas, and the results would be even more favourable if it had a better structure of specialization in the initial period.
\end{abstract}

KeYwords | Technological Structure; Environmental Innovations; Climate Change; Technological Specialization

JEL CODES | O33; O34; Q54; Q55 


\section{Introdução}

O processo de mudanças climáticas resultante do acúmulo de gases de efeito estufa (GEE) na atmosfera é reconhecido como um dos maiores desafios da sociedade atual e o seu estudo constitui uma das principais orientaçōes no debate sobre a sustentabilidade. Os compromissos assumidos no Protocolo de Kyoto ${ }^{1}$ evidenciaram a necessidade de estabilização das emissões globais de GEE e têm gerado consequências, tais como o aumento da demanda por novas tecnologias e a melhoria substancial das existentes (ARROW et al., 2009; MOWERY et al., 2010).

Enquanto a maioria das tecnologias de mitigação das mudanças climáticas tem sido concebida e utilizada por nações desenvolvidas, o aumento das projeçôes de emissões de GEE oriundas de economias emergentes tem chamado a atenção da literatura sobre como tais países estão sendo envolvidos nesse processo e como as transformações no âmbito internacional têm afetado seus perfis de especialização tecnológica.

Neste cenário o Brasil é um importante ator. Além do seu papel como potência econômica emergente, ao longo das últimas décadas o país passou a ser reconhecido internacionalmente por seu desempenho em áreas com potencial para mitigação das mudanças climáticas. O processo de abertura econômica dos anos 1990 resultou em reestruturação produtiva, atração de novas empresas e mudanças na sua competitividade industrial, com importantes repercussões sobre a sua estrutura tecnológica. Ainda que importantes estudos tenham sido desenvolvidos, existem lacunas a serem preenchidas, especialmente no que se refere à análise das transformaçôes na estrutura tecnológica brasileira em setores relacionados às mudanças no clima.

O presente artigo tem como objetivo investigar a evolução da estrutura tecnológica brasileira em áreas com potencial de mitigação de mudanças climáticas no período pós-Kyoto. A partir da constituição de uma base de dados original com patentes relacionadas às mudanças climáticas, será possível interpretar as transformações nos perfis de especialização nesses setores e a evolução do seu desempenho tecnológico em comparação a países selecionados. Para além das limitações metodológicas impostas a esse trabalho, sua proposição é relevante por dois aspectos principais: primeiro, pela incipiência deste debate no contexto brasileiro, com relativamente poucas pesquisas e limitação de fontes de dados; e, segundo, em função da importância crescente

1 O presente trabalho segue a tendência de uma extensa literatura que considera o processo de negociação e ratificação desse tratado internacional de compromissos de redução de GEE, ocorrido entre 1997 e 1999, como um marco analítico de diversas transformaçōes relacionadas às mudanças climáticas. 
destas tecnologias, que são reconhecidas internacionalmente pelo seu potencial na mitigação e adaptação das mudanças climáticas. Esses aspectos garantem à proposta deste estudo destacada importância na compreensão destas transformações no contexto brasileiro e de sua inserção internacional.

$\mathrm{O}$ artigo está organizado em cinco seções, além desta introdução. A seguir são abordados os aspectos teóricos e conceituais das inovaçôes ambientais e da especialização tecnológica dos países. Posteriormente, apresentam-se as fontes de dados e a metodologia aplicada, bem como o perfil de especialização em tecnologias para mitigação das mudanças climáticas no Brasil. Discutem-se os resultados da aplicação do método de decomposição estrutural e, por fim, são tecidas as consideraçôes finais.

\section{Inovações ambientais e especialização tecnológica}

Nas duas últimas décadas, os acordos de emissões de GEE entre países, tendo como ponto de partida o Protocolo de Kyoto, têm contribuído para a transformação do contexto internacional, produzindo nova dinâmica para as inovações ambientais ${ }^{2}$ e promovendo mudanças no comportamento inovativo das empresas e alterações na estrutura da especialização tecnológica dos países nesses setores.

Em termos conceituais, a especialização tecnológica de um país é dada pela regularidade dos campos técnicos em que atua. Ao longo das últimas décadas, uma ampla literatura tem se dedicado a compreender esse processo e como ele é explicado a partir de fatores como os níveis de atividade inovadora, a estrutura de produção e os padrões de exportação. É considerado especializado em determinado campo técnico o país cujo desempenho é relativamente superior àquele observado no nível internacional (ARCHIBUGI; PIANTA, 1992, 1994; LAURSEN, 1999; MALERBA; MONTOBBIO, 2003).

Diversos estudos dedicam-se a encontrar evidências empíricas do processo de especialização tecnológica dos países. De forma geral, enquanto países economicamente pequenos tendem a se especializar em campos tecnológicos restritos, as grandes nações distribuem suas atividades em um amplo número de campos tecnológicos (MANCUSI, 2001, 2003). Enquanto grande parte da literatura demonstra

2 Uma inovação ambiental é conceitualmente entendida como uma "[...] inovação que ocorre em tecnologias ambientais ou processos de controle da emissão de poluentes ou alteração dos processos de produção para reduzir ou evitar emissôes" (STERN, 2007). No escopo deste artigo, tal conceito de inovação ambiental é percebido mais especificamente como aquele com potencial para mitigação das mudanças climáticas. Autores também utilizam o conceito de ecoinovação para definir as inovaçôes com potencial para redução dos impactos ambientais negativos resultantes das atividades produtivas (RENNINGS, 2000; PUJARI, 2006; KEMP; PEARSON, 2008; HORBACH et al., 2012). 
o aumento da especialização tecnológica nos anos 1970 e 1980, o recente estudo de Picci e Savorelli (2013) mostra sua redução nas duas últimas décadas.

Outra parte importante desta literatura tem buscado compreender as relações entre a especialização tecnológica e os padrões de comércio internacional, em países do Leste Asiático (UCHIDA; COOK, 2005), da América Latina (HUANG; MIOZZO, 2003) e em países em desenvolvimento selecionados (MONTOBBIO; RAMPA, 2005). Esses estudos também se estendem na compreensão das relações entre especialização e balanço de pagamentos (MELICIANI, 2002) e dos impactos sobre o crescimento econômico de longo prazo (JUNGMITTAG, 2004).

Sobre a relação entre especialização tecnológica e padrões setoriais de atividade inovativa, evidências internacionais revelam que esses padrões diferem sistematicamente entre as classes tecnológicas, ao mesmo tempo que são notavelmente similares entre países. Na determinação de padrões em classes específicas, os regimes tecnológicos constituem o fator explicativo mais relevante, ainda que não o único. Os regimes tecnológicos são um importante elemento teórico para análise da mudança técnica a partir de características como oportunidade, cumulatividade, condições de apropriabilidade e base de conhecimento. As oportunidades tecnológicas diferem setorialmente e, ainda que possam ser mais ricas em alguns segmentos do que em outros, são limitadas pelas janelas abertas nas mudanças dos paradigmas tecnológicos. As condições de apropriabilidade são garantidas pelo caráter público do conhecimento, sendo que os mecanismos de apropriação, ainda que estáveis ao longo do tempo, também variam em cada setor (KLEVORICK et al., 1995; MALERBA; ORSENIGO, 1996).

Se as oportunidades são abundantes e as condições de apropriabilidade favoráveis, as empresas terão fortes incentivos para investir nestas tecnologias. Por outro lado, se as oportunidades são pequenas e o grau de apropriabilidade baixo, pode ser necessária uma maior ação do governo apoiando pesquisas e gastos privados em P\&D. O grau de cumulatividade dos regimes pode favorecer empresas estabelecidas que já realizam essas inovações ao longo de uma trajetória tecnológica específica. Entretanto, se for preciso uma inovação radical, a existência de alto grau de cumulatividade é capaz de bloquear seu surgimento (FABER; FRENKEN, 2009).

A especialização tecnológica dos países é um importante aspecto dos padrões setoriais da sua atividade inovativa e a literatura tem buscado compreender de que maneira essas atividades são persistentes ao longo do tempo, por representar uma forma relevante de análise da evolução e da dinâmica industrial (MALERBA et al., 1997; CEFIS; ORSENIGO, 2001). As evidências relacionam a especialização 
tecnológica com a existência de um núcleo estável e persistente de firmas inovadoras, fortemente afetado pela direção de spillovers setoriais entre países (MALERBA; ORSENIGO, 1996; MALERBA; MONTOBBIO, 2003).

Essa persistência está fortemente relacionada com um processo gradual de acumulação tecnológica e estável ao longo do tempo. O caráter cumulativo condiciona os padrões de especialização no futuro às realizações tecnológicas do passado, ou seja, com forte componente path dependence. Em vários setores as firmas inovadoras de hoje também foram as inovadoras no passado, mas a natureza cumulativa e dependente da trajetória de mudança técnica é maior naquelas com gastos mais elevados em P\&D, concentração em inovação de produto e de grande porte. Entretanto, das firmas que inovam, poucas o fazem persistentemente. A sustentação de uma capacidade de inovação por períodos prolongados é complexa mesmo para as grandes firmas inovadoras, já que seu grau de persistência em atividades inovadoras diminui ao longo do tempo (CEFIS, 2003; ANTONELLI et al., 2012; ARCHIBUGI et al., 2013).

A análise da persistência em contraposição à heterogeneidade trouxe a discussão sobre a associação da especialização tecnológica dos países a um processo de "destruição criativa", de um lado, e "acúmulo criativo", de outro. ${ }^{3}$ Numa perspectiva extrema, a visão de "destruição criativa” percebe a especialização como um processo irregular e aleatório, impulsionado por firmas homogêneas em busca de oportunidades tecnológicas que são acessíveis a todas. Em contraste, a visão de "acúmulo criativo", ao enfatizar que o conhecimento tem um forte componente tácito e altamente específico, trata a especialização como resultado da acumulação interna de competências tecnológicas por parte de empresas heterogêneas (MALERBA et al., 1997, ARCHIBUGI et al., 2013)

Aplicadas à discussão deste artigo, as transformações impostas pelas mudanças climáticas também podem ser vistas em ambas as óticas. $\mathrm{Na}$ visão do "acúmulo criativo", a especialização ocorre de forma regular ao longo de uma trajetória tecnológica estabelecida e empresas realizam inovações ambientais como rotina para evitar a entrada de novos concorrentes. Já na visão da "destruição criativa”, a necessidade de desenvolver e aprimorar tecnologias que contribuam para a estabilização das mudanças climáticas promove transformações no perfil tecnológico de indústrias estabelecidas e novas firmas buscam a exploração de oportunidades emergentes.

3 Enquanto o processo de "destruição criativa" é vinculado ao "Schumpeter Mark I", assim conhecido como modelo interpretativo desenvolvido na obra A teoria do desenvolvimento econômico, a "acumulação criativa" é relacionada ao "Schumpeter Mark II", tal como a literatura rotula o modelo proposto na obra Capitalismo, socialismo e democracia. 
Como resultado, os países são submetidos a grandes transformações nos seus campos de especialização tecnológica.

\section{Descrição dos dados e metodologia}

\subsection{As bases de dados de patentes}

Os dados de patentes utilizadas neste trabalho foram extraídos do European Patent Office (EPO), por meio do Banco Mundial de Estatísticas de Patentes (PATSTAT), na sua edição de abril de 2012, e do Instituto Nacional de Propriedade Industrial (Inpi), a partir da primeira versão da Base de Dados sobre Propriedade Intelectual para fins Estatísticos (Badepi). As patentes são percebidas neste trabalho como um indicador de mudança tecnológica no perfil competitivo setorial dos países em áreas relacionadas às mudanças climáticas. Suas estatísticas são fontes de informações adequadas às necessidades deste trabalho diante da sua homogeneidade, abrangência intertemporal e divisão baseada na Classificação Internacional de Patentes (IPC). ${ }^{4}$

Em cada uma destas bases foram selecionadas todas as patentes que possuem um ou mais campos técnicos (IPCs) que foram caracterizados pela Organização Mundial da Propriedade Intelectual (Ompi) como tecnologias para mitigação das mudanças climáticas. Entre as classificações empregadas na identificação destas patentes, utiliza-se o IPC Green Inventory, com nível de desagregação de três a dez dígitos. ${ }^{5}$ Tal classificação tem sido a principal forma para os escritórios de patentes em todo o mundo, incluindo o brasileiro, caracterizarem as denominadas "patentes verdes". Nestes escritórios os depósitos deste grupo têm sido examinados em ritmo mais rápido do que os pedidos de patente regulares, a partir do uso de procedimentos de fast-tracking (DECHEZLEPRÊTRE, 2013).

As patentes selecionadas foram organizadas em dois períodos de 12 anos - 1987-1998 e 1999-2010 -, a partir da sua data de depósito. Para os objetivos deste trabalho, tais amostras não diferenciam o tipo de depositante, de forma que as patentes podem ter sido depositadas por empresas, inventores individuais, universidades ou institutos de pesquisa. ${ }^{6}$ Uma síntese dos resultados encontrados é apresentada na Tabela 1.

4 Para uma análise dos dados de patentes como indicador de desempenho de inovação, ver Griliches (1990).

5 O chamado "IPC Green Inventory" foi desenvolvido por um comitê de especialistas, a fim de facilitar as buscas por informaçôes de patente relacionadas às tecnologias ambientalmente saudáveis (TAS), a partir das determinaçôes da Convenção Quadro das Naçôes Unidas sobre Mudanças Climáticas (UNFCCC).

6 Como já destacado em trabalhos como o de Albuquerque (2000), é importante ressaltar que no Brasil o peso de inventores individuais no total de patentes é significativamente elevado. 
TABELA 1

Número de classes (IPCs) e patentes, segundo setores relacionados às tecnologias para mitigação das mudanças climáticas

Brasil e Mundo - 1987-2010

\begin{tabular}{l|c|cc}
\hline \multirow{1}{*}{ Setores } & \multirow{2}{*}{$\begin{array}{c}\text { Número de } \\
\text { classes (IPC) }\end{array}$} & \multicolumn{2}{c}{$\begin{array}{c}\text { Número de patentes } \\
(\mathbf{1 9 8 7 - 2 0 1 0})\end{array}$} \\
\cline { 3 - 4 } & & \multicolumn{1}{c|}{ Brasil } & Mundo \\
\hline 1. Energias alternativas & 309 & 9.103 & 82.253 \\
2. Transportes & 108 & 1.031 & 12.836 \\
3. Conservação de energia & 81 & 1.333 & 24.577 \\
4. Gerenciamento de resíduos & 256 & 5.524 & 39.261 \\
5. Agricultura & 231 & 8.967 & 26.651 \\
6. Aspectos administrativos, & 24 & 64 & 72 \\
regulatórios e design & 5 & 3 & 58 \\
7. Energia nuclear & & $\mathbf{2 6 . 0 2 5}$ & $\mathbf{1 8 5 . 7 0 8}$ \\
Total & $\mathbf{1 . 0 1 4}$ & $\mathbf{2 6 5}$ \\
\hline
\end{tabular}

Fonte: EPO (2012); Inpi (2013). Elaboração do autor.

Entretanto, cabe reconhecer algumas limitações do artigo. De modo geral, os indicadores baseados em patentes são proxys imperfeitas de inovação tecnológica. As patentes constituem apenas uma das formas para proteção das invenções e os seus proprietários podem evitar o patenteamento por motivo de sigilo, impedindo sua divulgação pública, ou ainda para não incorrer nos custos do seu registro (COHEN et al., 2000). Por outro lado, são poucos os casos de invenções economicamente importantes que não foram patenteadas (DERNIS et al., 2001).

Outra limitação relevante deste trabalho é a existência de diferenças setoriais nas propensôes a patentear, que podem variar de acordo com os regimes tecnológicos. Em estudos que medem a atividade de patenteamento em diferentes países, tais diferenças também são causadas por variações no rigor com que os escritórios de patentes aplicam os direitos de propriedade intelectual. Dessa forma, uma maior atividade de patenteamento pode refletir tanto uma maior atividade inventiva quanto uma maior propensão à concessão de patentes (COHEN et al., 2000). Neste trabalho, as metodologias propostas e o uso do EPO como escritório internacional de patentes resolvem parte deste problema. Também buscou-se controlar os problemas identificados por van Zeebroeck et al. (2006) quanto à sensibilidade da medição da especialização tecnológica com dados de patentes no que se refere à fonte de dados e ao nível de agregação setorial. 
Sobre o uso destas bases, na quarta seção, ao tratar das transformações no perfil de especialização tecnológica brasileira, utiliza-se a amostra extraída do Inpi, comparativamente às patentes mundiais depositadas no EPO. Já na análise da dinâmica internacional e da evolução da sua quota internacional, da quinta seção, emprega-se apenas a amostra extraída da base do EPO, diferenciando os depositantes pelo seu país de residência.

Em ambas as análises, a escolha do EPO justifica-se pela necessidade de construir uma representação qualitativa das patentes depositadas nesses setores em todo o mundo. O EPO é a mais comum alternativa de depósito para inventores fora da União Europeia. Na medida em que uma patente é concedida no escritório do país de origem do inventor, em grande parte dos casos, o EPO representa a primeira alternativa de proteção internacional (GRUPP; SCHOMACH, 1999). Ainda que não exista um consenso na literatura sobre qual escritório é mais representativo das patentes depositadas em todo o mundo, entende-se que o EPO possui algumas vantagens em relação ao Uspto (United States Patent and Trademark Office). Enquanto as patentes depositadas no EPO são extensíveis aos países membros da Convenção de Munique, ${ }^{7}$ a proteção dos depósitos realizados no Uspto restringe-se ao território norte-americano. Os custos dos depósitos no EPO são relativamente mais elevados e atuam como filtro econômico das patentes de baixo valor industrial. Por fim, as patentes concedidas pelo EPO são publicadas 18 meses após sua solicitação, contra um prazo médio de publicação de dois anos do Uspto (URRACA-RUIZ, 2013) ${ }^{8}$.

\subsection{A mensuração da especialização tecnológica e da decomposição estrutural}

Para cada uma destas análises são aplicadas diferentes metodologias. $\mathrm{Na}$ análise das transformações dos perfis de especialização tecnológica, realizada mais adiante na quarta seção, o presente estudo segue uma longa tradição empírica iniciada por Soete (1987), que considera as patentes uma proxy de força tecnológica do país e propõe a aplicação do indicador de vantagem comparativa revelada de Balassa (1965) para estimar um índice de vantagem tecnológica revelada (VTR) do país $c$ no campo tecnológico $j$, que pode ser algebricamente apresentado da seguinte forma:

7 A Convenção Europeia de Patentes, enquanto um tratado multilateral, harmoniza o processo de concessão e garante proteção patentária aos países signatários por meio de um sistema jurídico autônomo.

8 Outros estudos sistematizados por Urraca (2008) reforçam a tese de menor internacionalização do Uspto diante da pequena participação de inventores não residentes. 


$$
V T R_{c j}=\frac{p_{c j}}{\sum_{c} p_{c j}} / \frac{\sum_{j} p_{c j}}{\sum_{j} \sum_{c} p_{c j}}
$$

Onde $p_{c j}$ é o total de patentes do país $c$ no campo $j ; \sum_{c} p_{c j}$ compreende o somatório de patentes depositadas pelo país $c ; \sum_{j} p_{c j}$ corresponde à soma de todas as patentes mundiais do campo $j$; e $\sum_{j} \sum_{c} p_{c j}$ é o total de patentes mundiais. Nessas condições, quando é encontrado um valor de VTR superior a 1, entende-se que se trata de um campo técnico em que há especialização.

A especialização tecnológica está relacionada às vantagens relativas e não absolutas. Em princípio, um país pode ter vantagens absolutas (e ser mais inovador) em todas as tecnologias, porém, mesmo neste caso, tem vantagens em relação a algumas tecnologias em comparação com as outras. Dessa forma, a força tecnológica de um país em uma tecnologia específica pode ser medida pela proporção de patentes daquele país para o total mundial de patentes naquela tecnologia.

Entende-se como nível de oportunidade tecnológica (OT) a participação de determinado campo técnico no total de patentes mundiais em ambos os períodos. Já o grau da OT refere-se à variação, entre os períodos analisados, da participação no total de patentes mundiais, de forma que são positivos os campos que ganharam participação no total e negativos aqueles que perderam. Enquanto o nível de OT é capaz de indicar os campos que possuem maior apropriabilidade, o grau de OT revela os campos em que existiu maior possibilidade de capturar oportunidades entre os períodos.

Em busca de uma melhor compreensão da dinâmica internacional de patenteamento e dos fatores que condicionam a variação na participação internacional nessas tecnologias, a quinta seção deste artigo apresenta os resultados da aplicação do método de análise de decomposição estrutural, originalmente proposto por Fagerberg e Sollie (1987), também conhecido como Constant Market Share (CMS). Esse método é utilizado tanto em estudos empíricos sobre o comércio internacional como naqueles de dinâmica tecnológica (LAURSEN, 1999; MONTOBBIO; RAMPA, 2005; URRACA-RUIZ, 2008, 2013). Sua vantagem é proporcionar o isolamento do efeito da mudança estrutural e a decomposição da variação da participação de patentes entre períodos. Portanto, a taxa de variação da participação de determinado país sobre o total de patentes mundiais $\left(\Delta p_{j}\right)$ é decomposta em três efeitos principais, como segue. 


$$
\Delta p_{j}=\sum_{i}\left(\Delta p_{i j} o_{i}^{t-1}\right)+\sum_{i}\left(p_{i j}^{t-1} \Delta o_{i}\right)+\sum_{i}\left(\Delta p_{i j} \Delta o_{i}\right)
$$

Onde $p_{j}=\sum_{i} P_{i j} / \sum_{i} \sum_{j} P_{i j}$ é a participação agregada do país $j$ no total mundial de patentes; $p_{i j}=P_{i j} / \sum_{j} P_{i j}$ refere-se à participação de um dado campo $i$ em termos de patentes; e $o_{i}=\sum_{i} P_{i j} / \sum_{i} \sum_{j} P_{i j}$ corresponde à participação do setor $i$ no total de patentes.

O efeito "technology share" (i) permite afirmar se a variação é justificada pelo fato de o país ter ganhado participação em patenteamento, assumindo que a estrutura é a mesma nos dois períodos em questão. O efeito "structural technology" (ii) relaciona a variação com um "correto" (“ou errado”) padrão de especialização inicial e o efeito "technology adaptation" (iii) avalia o movimento de entrada ou saída em setores com rápido crescimento (ou estagnação) da atividade tecnológica. Para avaliar se o efeito "technology adaptation" é de entrada nos setores "corretos" ou de saída dos setores "errados", é necessária sua decomposição em dois outros efeitos, que são "technology growth adaptation" (iv), que será positivo se houve um movimento para setores de rápido crescimento, e "technology stagnation adaptation” (v), que será positivo se ocorreu uma saída dos setores estagnados, expressos da seguinte forma:

$$
\sum_{i}\left(\Delta p_{i j} \Delta o_{i}\right)=\sum_{i}\left(\Delta p_{i j}\left(\Delta o_{i}+\left|\Delta o_{i}\right|\right) / 2\right)+\sum_{i}\left(\Delta p_{i j}\left(\Delta o_{i}-\left|\Delta o_{i}\right|\right) / 2\right)
$$

Portanto, a análise completa da decomposição estrutural pode ser escrita da seguinte maneira:

$$
\Delta p_{j}=\sum_{i}\left(\Delta p_{i j} o_{i}^{t-1}\right)+\sum_{i}\left(p_{i j}^{t-1} \Delta o_{i}\right)+\sum_{i}\left(\Delta p_{i j}\left(\Delta o_{i}+\left|\Delta o_{i}\right|\right) / 2\right)+\sum_{i}\left(\Delta p_{i j}\left(\Delta o_{i}-\left|\Delta o_{i}\right|\right) / 2\right)
$$

Dessa forma, os resultados encontrados por meio dos efeitos "structural technology" e "technology adaptation" revelam os movimentos de entrada e saída dos países em setores com diferentes oportunidades tecnológicas. Caso esses dois efeitos apresentem valores altos e positivos, pode-se afirmar que esse país se moveu para os setores com elevadas oportunidades tecnológicas e abandonou setores estagnados. 
Para os objetivos deste trabalho, o crescimento relativo do número de patentes de determinada classe no mundo é percebido como uma proxy de oportunidade tecnológica. Assim, o aumento do número de patentes de um setor é capaz de indicar se o crescimento da sua OT é relativamente alto ou baixo. Essa variação também pode ser vista como o acesso de determinado país a setores com níveis relativamente altos de oportunidade tecnológica (CANTWELL; ANDERSEN, 1996; LAURSEN, 1999). Em um estudo aplicado, Nelson e Wolff (1997) mostraram que os níveis de oportunidades tecnológicas também guardam significativa relação com os níveis de P\&D.

\section{As especializações brasileiras em tecnologias para mitigação das mudanças climáticas}

Podem-se identificar importantes estudos sobre o Brasil no período recente, com abordagens próximas à proposta neste artigo. O trabalho de Urraca-Ruiz (2008), ao avaliar o impacto da abertura comercial na estrutura tecnológica, mostrou que o país foi capaz de ampliar de sete para 11 a quantidade de campos em que detém especialização tecnológica, demonstrando certo dinamismo na criação de competências, dada a capacidade de diversificar sua base técnica e aproveitar a abertura de janelas de oportunidade. Contudo, esse dinamismo não foi capaz de viabilizar o ingresso em setores com maiores oportunidades tecnológicas, mantendo-se, consequentemente, não especializado em setores-chave como eletrônica, eletricidade, instrumentos ou máquinas e ferramentas.

Sobre as tecnologias mais promissoras na mitigação das mudanças climáticas no Brasil, o trabalho de Porto et al. (2012) mostra que a busca pela diminuição do gap em relação à fronteira tecnológica resultou no acúmulo de um conjunto pulverizado de competências em tecnologias ambientais e, em todos os setores, o registro de um percentual elevado de patentes isoladas, ou seja, que provocam poucos desdobramentos e que se supõe ter impacto pontual.

Ao analisar oito campos relacionados às tecnologias ambientais, Urraca-Ruiz e Durán-Romero (2013) mostram que 76,5\% das patentes com inventores com sede no Brasil são depositadas por agentes não residentes, das quais 69,3\% são multinacionais não residentes no Brasil, o que representa um forte indício de que as competências locais nessas tecnologias são capturadas por multinacionais não residentes. Nesse contexto, o país ainda observa um baixo nível de esforço em P\&D realizado por empresas nacionais e por multinacionais com sede no país. 
Os resultados do trabalho de Lucchesi (2013) sobre os determinantes da inovação ambiental na indústria de transformação brasileira e seus efeitos sobre a demanda de mão de obra e o valor adicionado vão ao encontro das evidências internacionais e reforçam o papel da regulamentação ambiental como elemento indutor da adoção dessas inovaçôes.

Sobre as estratégias inovativas das empresas brasileiras, Queiroz e Podcameni (2014) encontram significativas relações entre a introdução de inovações ambientais e o tamanho e a origem estrangeira do capital destas empresas, o que evidencia a importância da inserção internacional. Seus resultados finais afirmam que os esforços inovativos não convergem para as questôes ambientais por serem focados em elementos que têm pouca ou nenhuma relação com inovações ambientais.

Entretanto, não há estudos que avaliem mais detalhadamente as alterações nos perfis de especialização em tecnologias para mitigação das mudanças climáticas no Brasil. Com esse objetivo, são apresentadas na Tabela 2 as estimativas das vantagens tecnológicas por área e setor, bem como seu grau e nível de oportunidade tecnológica (OT).

Os maiores níveis de OT, em todo o período, foram registrados pelos setores energias alternativas e gerenciamento de resíduos e pelas áreas de biocombustíveis e controle da poluição. Entretanto, mesmo que haja um elevado nível de OT, como nos setores de energias alternativas, gerenciamento de resíduos e agricultura, pode não ocorrer variação positiva no seu grau de OT. Das 39 áreas analisadas, 19 registraram aumento do grau de OT, cinco se mantiveram estáveis e 15 perderam participação e apresentaram baixo grau de OT. Das áreas com grau de OT positivo, as maiores variaçôes foram observadas em dispositivos para produção de energia mecânica a partir de energia muscular, energia eólica, células-combustível e iluminação de baixa energia.

Sobre as transformaçōes nos perfis de especialização dos setores entre os períodos analisados, observa-se que o Brasil foi capaz de manter sua especialização em aspectos administrativos, regulatórios ou design e agricultura. Entretanto, quando se analisam as áreas tecnológicas, percebe-se que o país era especializado em 15, perdeu especialização em seis áreas e ingressou em outras duas. Na seção seguinte é apresentada uma proposta de interpretação setorial dos resultados encontrados, com o objetivo de contextualizar o debate. Essas interpretações têm caráter ilustrativo e seu nível de detalhamento é restringido pelo nível de agregação adotado e potencial da metodologia empregada. 


\section{TABELA 2}

Estimativas das vantagens tecnológicas reveladas (VTR) e grau e nível de oportunidade tecnológica (OT), segundo setores e áreas

Brasil - 1987-2010

\begin{tabular}{|c|c|c|c|c|c|c|}
\hline \multirow[b]{2}{*}{ Cod. } & \multirow[b]{2}{*}{ Setores e áreas } & \multicolumn{3}{|c|}{ VTR } & \multirow{2}{*}{$\begin{array}{c}\Delta \text { Grau } \\
\text { OT }\end{array}$} & \multirow{2}{*}{$\begin{array}{c}\text { Nível } \\
\text { OT } \\
(\%)\end{array}$} \\
\hline & & $\begin{array}{l}1987- \\
1998\end{array}$ & $\begin{array}{l}1999- \\
2010\end{array}$ & $\begin{array}{c}\Delta \text { VTR } \\
(\%)\end{array}$ & & \\
\hline 1.0 & Energias alternativas & 0,67 & 0,68 & 2,21 & $-0,09$ & 3,21 \\
\hline 1.1 & Biocombustíveis & 0,60 & 0,87 & 46,11 & $-0,44$ & 1,39 \\
\hline 1.2 & $\begin{array}{l}\text { Ciclo combinado de gaseificação integrada } \\
\text { (IGCC) }\end{array}$ & 1,06 & 0,91 & $-13,65$ & $-0,37$ & 0,02 \\
\hline 1.3 & Células-combustível & 0,38 & 0,38 & $-0,55$ & 1,74 & 0,51 \\
\hline 1.4 & Pirólise ou gaseificação de biomassa & 0,67 & 1,07 & 59,46 & $-0,69$ & 0,01 \\
\hline 1.5 & $\begin{array}{l}\text { Aproveitamento de energia a partir de } \\
\text { resíduos de atividades humanas }\end{array}$ & 0,98 & 0,96 & $-1,21$ & $-0,35$ & 0,39 \\
\hline 1.6 & Energia hidráulica & 3,04 & 1,64 & $-46,05$ & 1,47 & 0,03 \\
\hline 1.7 & $\begin{array}{l}\text { Conversão da energia térmica dos oceanos } \\
\text { (OTEC) }\end{array}$ & 1,38 & 0,81 & $-41,42$ & $-0,04$ & 0,00 \\
\hline 1.8 & Energia eólica & 1,82 & 0,84 & $-53,73$ & 1,89 & 0,06 \\
\hline 1.9 & Energia solar & 0,56 & 0,49 & $-12,00$ & 0,71 & 0,58 \\
\hline 1.10 & Energia geotérmica & 1,02 & 0,47 & $-54,39$ & 0,38 & 0,05 \\
\hline 1.11 & $\begin{array}{l}\text { Outros tipos de produção ou utilização de } \\
\text { calor }\end{array}$ & 0,51 & 0,59 & 16,17 & 0,16 & 0,02 \\
\hline 1.12 & Utilização de calor residual & 0,71 & 0,53 & $-25,31$ & $-0,04$ & 0,14 \\
\hline 1.13 & $\begin{array}{l}\text { Dispositivo para produção de energia } \\
\text { mecânica a partir de energia muscular }\end{array}$ & 16,60 & 3,24 & $-80,47$ & 5,70 & 0,00 \\
\hline 2.0 & Transportes & 0,71 & 0,42 & $-41,73$ & 0,45 & 0,50 \\
\hline 2.1 & Veículos em geral & 0,69 & 0,41 & $-40,36$ & 0,48 & 0,46 \\
\hline 2.2 & Veículos exceto veículos ferroviários & 1,19 & 0,50 & $-57,76$ & 0,27 & 0,03 \\
\hline 2.3 & Veículos ferroviários & 2,55 & 0,25 & 0,00 & $-0,04$ & 0,00 \\
\hline 2.4 & Propulsão de embarcação marítima & 4,15 & 2,47 & $-40,49$ & 0,26 & 0,00 \\
\hline 2.5 & Veículos que utilizam a energia solar & 0,24 & 0,15 & $-39,44$ & $-0,38$ & 0,01 \\
\hline 3.0 & Conservação de energia & 0,48 & 0,29 & $-39,34$ & 0,66 & 0,96 \\
\hline 3.1 & Armazenagem de energia elétrica & 0,52 & 0,36 & $-30,95$ & 0,58 & 0,23 \\
\hline 3.2 & $\begin{array}{l}\text { Circuitos de alimentação de energia } \\
\text { elétrica }\end{array}$ & 1,61 & 0,38 & $-76,50$ & 0,66 & 0,01 \\
\hline 3.3 & Medição do consumo de eletricidade & 0,57 & 0,47 & $-16,67$ & 0,74 & 0,02 \\
\hline 3.4 & Armazenamento de energia térmica & 0,86 & 0,66 & $-22,75$ & 0,12 & 0,04 \\
\hline 3.5 & Iluminação de baixo consumo energético & 0,10 & 0,13 & 33,21 & 1,56 & 0,45 \\
\hline 3.6 & Isolamento térmico de edificaçōes & 0,71 & 0,50 & $-28,85$ & $-0,12$ & 0,19 \\
\hline 3.7 & Recuperação de energia mecânica & 1,16 & 1,74 & 49,52 & 0,69 & 0,01 \\
\hline 4.0 & Gerenciamento de resíduos & 0,87 & 0,85 & $-3,09$ & $-0,21$ & 1,53 \\
\hline 4.1 & Eliminação de resíduos & - & - & - & - & - \\
\hline
\end{tabular}


TABELA 2

Estimativas das vantagens tecnológicas reveladas (VTR) e grau e nível de oportunidade tecnológica (OT), segundo setores e áreas

Brasil - 1987-2010

(continuação)

\begin{tabular}{|c|c|c|c|c|c|c|}
\hline \multirow[b]{2}{*}{ Cod. } & \multirow[b]{2}{*}{ Setores e áreas } & \multicolumn{3}{|c|}{ VTR } & \multirow[b]{2}{*}{$\begin{array}{c}\Delta \text { Grau } \\
\text { OT }\end{array}$} & \multirow{2}{*}{$\begin{array}{r}\text { Níve } \\
\text { OT } \\
(\%)\end{array}$} \\
\hline & & $\begin{array}{l}1987- \\
1998\end{array}$ & $\begin{array}{l}1999- \\
2010\end{array}$ & $\begin{array}{c}\Delta \text { VTR } \\
(\%)\end{array}$ & & \\
\hline 4.2 & Tratamento de resíduos & 0,70 & 0,85 & 20,87 & $-0,66$ & 0,08 \\
\hline 4.3 & Destruição de resíduos por combustão & - & - & - & - & - \\
\hline 4.4 & Reutilização de materiais usados & 1,12 & 1,63 & 46,48 & $-0,48$ & 0,19 \\
\hline 4.5 & Controle de poluição & 0,84 & 0,75 & $-10,82$ & $-0,11$ & 1,26 \\
\hline 5.0 & Agricultura & 1,91 & 2,14 & 11,67 & $-0,32$ & 1,04 \\
\hline 5.1 & Técnicas de reflorestamento & 2,28 & 2,02 & $-11,47$ & 0,27 & 0,00 \\
\hline 5.2 & Técnicas alternativas de irrigação & 4,64 & 1,85 & $-60,09$ & $-0,03$ & 0,00 \\
\hline 5.3 & Pesticidas alternativos & 1,90 & 2,14 & 12,85 & $-0,32$ & 1,03 \\
\hline 5.4 & Melhoria do solo & 2,72 & 1,78 & $-34,62$ & $-0,80$ & 0,01 \\
\hline 6.0 & $\begin{array}{l}\text { Aspectos administrativos, regulatórios } \\
\text { ou design }\end{array}$ & 9,75 & 3,43 & $-64,79$ & 0,16 & 0,00 \\
\hline 6.1 & $\begin{array}{l}\text { Deslocamentos, ex. faixa exclusiva, } \\
\text { trabalho remoto, etc. }\end{array}$ & - & - & - & - & - \\
\hline 6.2 & $\begin{array}{l}\text { Carbono/comércio de emissões, ex. } \\
\text { créditos de poluição }\end{array}$ & - & - & - & - & - \\
\hline 6.3 & Design de estática nas estruturas & 0,00 & 3,43 & 0,00 & 0,16 & 0,00 \\
\hline 7.0 & Energia nuclear & 0,55 & 0,27 & $-51,18$ & 1,30 & 0,00 \\
\hline 7.1 & Engenharia nuclear & - & - & - & - & - \\
\hline 7.2 & $\begin{array}{l}\text { Usinas de turbina a gás com fonte de } \\
\text { calor de origem nuclear }\end{array}$ & 0,00 & 0,27 & 0,00 & 1,30 & 0,00 \\
\hline
\end{tabular}

Fonte: EPO (2012); Inpi (2013). Elaboração do autor.

\subsection{Análise das transformações dos perfis setoriais}

O setor de energias alternativas possui o maior nível de oportunidade tecnológica entre os setores analisados, ainda que a variação no grau de OT tenha sido praticamente nula $(-0,09)$. Entre as áreas que compóem esse setor, o Brasil manteve sua especialização em energia hidráulica - área tecnologicamente madura no país -, dispositivos para produção de energia mecânica a partir de energia muscular, além de ter se especializado em pirólise ou gaseificação de biomassa, resultado que pode ser visto como possível efeito dos investimentos para acompanhar a fronteira internacional.

Ainda nesse setor, observa-se a perda de especialização em ciclo combinado de gaseificação integrada (IGCC), conversão da energia térmica dos oceanos (OTEC), 
energia eólica e energia geotérmica. ${ }^{9}$ Sobre as áreas em que o país se manteve não especializado entre os períodos, a de biocombustíveis chama a atenção, já que o país é reconhecido por possuir capacitações acumuladas e uma importante tradição de pesquisa. ${ }^{10}$ As demais áreas são: células-combustível; aproveitamento de energia a partir de resíduos de atividades humanas; energia solar; outros tipos de produção ou utilização de calor; e utilização de calor residual. Em muitas dessas áreas, como energia eólica e energia solar (especificamente fotovoltaica), a ausência de especialização relaciona-se com o pequeno esforço tecnológico nacional para o seu desenvolvimento e as políticas adotadas que se concentram na atração de investimento estrangeiro direto.

O setor transportes é percebido como estratégico pelo potencial de mitigação de emissões de GEE no contexto brasileiro e pelo conjunto de tecnologias que têm se tornado disponíveis comercialmente. Ainda que o grau das oportunidades tecnológicas em todo o mundo tenha aumentado entre os períodos $(0,45)$, o país diminuiu sua vantagem tecnológica nesse setor e apenas na área de propulsão de embarcação marítima manteve sua especialização. As áreas veículos exceto veículos ferroviários e veículos ferroviários deixaram de ser especializadas e as de veículos em geral e veículos que utilizam a energia solar mantiveram-se não especializadas.

Analisando o setor conservação de energia, percebe-se o aumento da sua participação em todo o mundo, dada sua variação positiva no grau de oportunidade tecnológica $(0,66)$. No Brasil, não existe especialização na maioria dessas áreas, com exceção de circuitos de alimentação de energia elétrica, em que o país era especializado no período inicial, e recuperação de energia mecânica, na qual manteve sua especialização em ambos os períodos.

O setor gerenciamento de residuo diminuiu o seu grau de oportunidade entre os períodos analisados, entretanto, ainda constitui o segundo setor com maior nível de oportunidade tecnológica $(-0,21)$. No Brasil, verificaram-se, em ambos os períodos, a especialização na área reutilização de materiais usados e a manutenção da não especialização nas demais áreas.

Já o setor agricultura é bastante peculiar, tendo em vista que o Brasil manteve sua especialização em todas as áreas, mesmo em um contexto internacional de redução do grau de oportunidades tecnológicas $(-0,32)$. Neste setor, formado

9 Essas análises precisam ser vistas à luz das limitaçôes da metodologia empregada e devem reconhecer a possibilidade de as patentes selecionadas variarem no grau de correspondência com as tecnologias analisadas.

10 Complementar a este resultado, o estudo de Porto et al. (2013) mostra que o país incorre em riscos de dependência tecnológica em setores como etanol e biodiesel por não haver tecnologia nacional sendo desenvolvida em importantes rotas tecnológicas. 
por tecnologias relacionadas a reflorestamento, irrigação, pesticidas alternativos e melhoria do solo, o país sustentou todas as suas especializações entre os períodos. Esse resultado pode ser analisado no contexto da atração internacional exercida pelo setor, da longa tradição de pesquisa estabelecida no país e do papel central de instituiçôes envolvidas no desenvolvimento tecnológico.

O setor aspectos administrativos, regulatórios ou design é relativamente novo e estratégico no contexto da adaptação às mudanças climáticas. O país apresentou uma especialização na área design de estática nas estruturas, entretanto, esse resultado também precisa considerar a possibilidade de as patentes selecionadas terem uma menor correspondência com as tecnologias que se procura analisar.

Por fim, o setor de energia nuclear também registrou aumento no grau de oportunidades tecnológicas entre os períodos $(1,30)$, mas não existem especializações nas áreas estudadas e os resultados existentes são justificados por esforços empreendidos por instituições como a Comissão Nacional de Energia Nuclear (CNEN).

\subsection{Mobilidade e persistência da especialização}

Para analisar a dinâmica de ingresso e abandono de áreas de especialização entre os períodos, considera-se que $A_{i}$ seja o número de áreas em que o Brasil era especializado no período inicial, $A_{f}$ corresponda ao número de áreas nas quais era especializado no período final, e $A_{i f}$ refira-se às áreas em que a especialização ocorreu em ambos os períodos, para estimar um índice de persistência definido como $I P=A_{i f} / A_{t} \mathrm{e}$ um índice de mobilidade estimado por $I M=1-I P$, assumindo que $A_{t}=A_{i f}+A_{i}$ $+A_{f}$. Ambos os índices variam entre 0 e 1 e, caso o IP seja igual a 0 e o IM igual a 1 , significa que o país perdeu todas as especializações que havia criado no início do período. Do contrário, se forem registrados um IP igual a 1 e um IM igual a 0 , significa que o país manteve a mesma estrutura de especialização verificada no início do período.

Desse modo, entre os períodos analisados, o Brasil registrou um índice de persistência de 0,5294 e, consequentemente, um índice de mobilidade de 0,4706, sendo capaz de sustentar máxima persistência apenas nos setores gerenciamento de resíduo e agricultura. Contribuíram para uma elevada mobilidade os setores energia alternativa, transporte, conservação de energia e aspectos administrativos, regulatórios e design. 
Para uma maior compreensão dos níveis de persistência, testa-se em que medida a especialização tecnológica alcançada relaciona-se com o crescimento da sua quota de participação. Para tanto, calculam-se os coeficientes de correlação da VTR dos períodos inicial e final com a taxa de variação da participação no total de patentes por cada área $\left(\triangle p_{i j}\right)$. Essa correlação revela a proximidade da vantagem tecnológica com a variação na sua participação no total de patentes. Enquanto uma correlação positiva entre aumento na participação e vantagem final significa que a trajetória da atividade inovadora determina a especialização, a correlação positiva entre o aumento da participação e a vantagem inicial indica um determinismo na direção da mudança tecnológica, ou seja, a existência de cumulatividade (URRACA-RUIZ, 2008).

A partir dos resultados apresentados na Tabela 3, observa-se que em quatro dos sete setores a vantagem final se relaciona positivamente com o crescimento da sua participação $\left(\triangle p_{i j}\right)$, sendo que esse coeficiente é particularmente elevado no setor agricultura. Nesses setores, o crescimento da sua participação determinou as vantagens alcançadas no período final. Por outro lado, o setor energias alternativas foi o único em que o aumento desta participação esteve positivamente relacionado com a sua vantagem no período inicial, o que permite concluir que nesse setor a cumulatividade é um importante fator explicativo. Na próxima seção, busca-se uma maior compreensão da dinâmica de depósitos internacionais de patentes realizados por residentes a partir das análises de decomposição estrutural.

\section{TABELA 3}

Coeficientes de correlação entre vantagem tecnológica revelada (VTR) inicial e final e $\triangle p i j$, segundo setores

Brasil - 1987-2010

\begin{tabular}{l|c|c}
\hline \multirow{2}{*}{\multicolumn{1}{c}{ Setores }} & \multicolumn{2}{c}{ Coeficientes de correlação } \\
\cline { 2 - 3 } & $V T R_{1987-1998^{-\triangle p i j}} V T R_{1999-2010^{-\triangle p i j}}$ \\
\hline 1. Energias alternativas & 0,0988 & $-0,0254$ \\
2. Transportes & $-0,0005$ & 0,1077 \\
3. Conservação de energia & $-0,5308$ & 0,1916 \\
4. Gerenciamento de resíduos & $-0,6185$ & $-0,5752$ \\
5. Agricultura & $-0,4903$ & 0,8006 \\
6. Aspectos administrativos, & 0,0000 & 0,5000 \\
regulatórios e design & - & - \\
7. Energia nuclear & - & \\
\hline
\end{tabular}

Fonte: EPO (2012); Inpi (2013). Elaboraçăo do autor. 


\section{A dinâmica internacional e o desempenho brasileiro}

A partir desta seção, analisa-se a posição brasileira diante das transformações ocorridas na dinâmica internacional de patenteamento em tecnologias para mitigação das mudanças climáticas. De modo agregado, o número absoluto de patentes mundiais com campos técnicos relacionados à mitigação das mudanças climáticas cresceu 95,6\% entre os períodos analisados, entretanto, sua participação no total de depósitos se manteve relativamente estável ao longo dos anos, como é apresentado no Gráfico 1.

\section{GRÁFICO 1}

Participação de patentes de mitigação das mudanças climáticas no total de patentes Mundo - 1987-2010

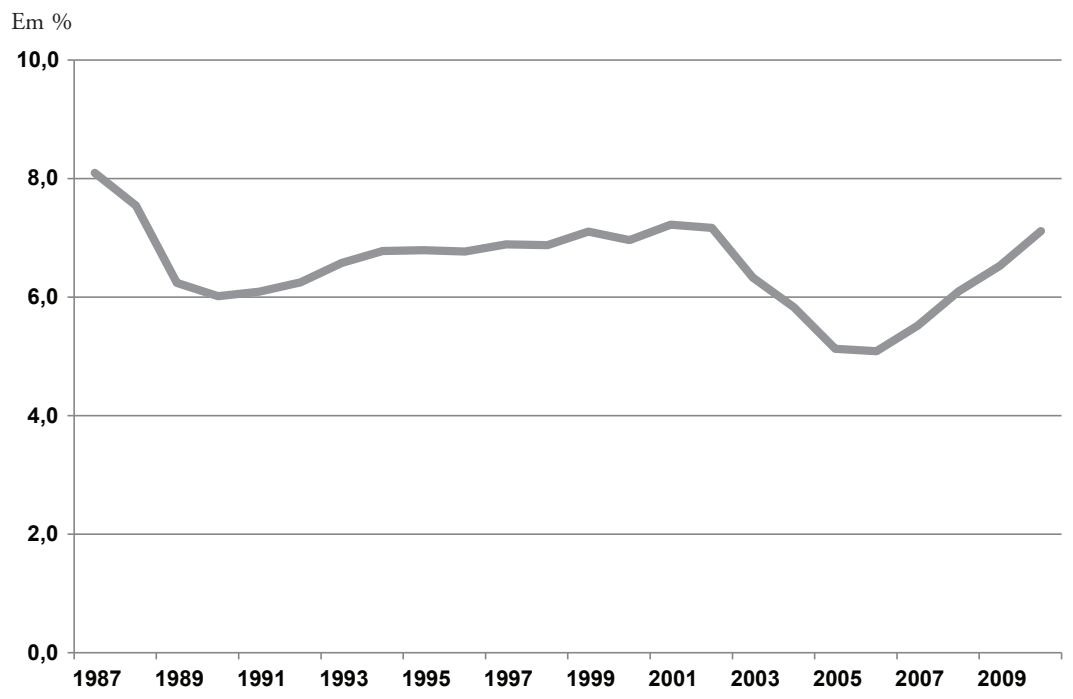

Fonte: EPO (2012). Elaboração do autor.

O depósito de patentes neste grupo de tecnologias no âmbito internacional tem forte concentração e é realizado majoritariamente por Estado Unidos, Alemanha e Japão, que responderam por $69,6 \%$ do total mundial. Resultado equivalente foi encontrado por Dechezleprêtre et al. (2011) que, ao examinarem as patentes depositadas em 13 campos tecnológicos relacionados às mudanças climáticas, mostraram que essas três naçôes representam dois terços de todo o esforço mundial.

Para além destes resultados, importantes mudanças foram observadas no período recente, a julgar pelo fato de todos os cinco maiores patenteadores, com exceção do Japão, terem diminuído sua participação no total de patentes mundiais. 
Essa dinâmica pode ser mais bem compreendida a partir do aumento da quota de diversos países, com destaque para Coreia do Sul, Taiwan, Rússia, China e Brasil.

Para interpretar os fatores responsáveis pela variação da participação na atividade inventiva, aplica-se o método empírico de decomposição estrutural que sistematiza esses resultados em termos de quatro efeitos principais. Os resultados são apresentados na Tabela 4.

\section{TABELA 4}

Resultados da decomposição estrutural por setores e áreas entre os períodos analisados Países selecionados - 1987-2010

\begin{tabular}{|c|c|c|c|c|c|c|c|c|}
\hline \multirow[b]{3}{*}{ Cod. } & \multirow[b]{3}{*}{ Países } & \multicolumn{2}{|c|}{$p i(\%)$} & \multicolumn{5}{|c|}{ Efeitos } \\
\hline & & \multirow[b]{2}{*}{$\begin{array}{c}1987- \\
1998\end{array}$} & \multirow[b]{2}{*}{$\begin{array}{l}1999- \\
2010\end{array}$} & \multirow[b]{2}{*}{$\begin{array}{c}\text { Tech } \\
\text { change }\end{array}$} & \multirow[b]{2}{*}{$\begin{array}{l}\text { Tech } \\
\text { share }\end{array}$} & \multirow{2}{*}{$\begin{array}{c}\text { Struc- } \\
\text { tural } \\
\text { tech }\end{array}$} & \multicolumn{2}{|c|}{ Adaptation } \\
\hline & & & & & & & $\begin{array}{c}\text { Tech } \\
\text { growth }\end{array}$ & $\begin{array}{c}\text { Tech } \\
\text { stag- } \\
\text { nation }\end{array}$ \\
\hline US & Estados Unidos & 30,55 & 22,87 & $-25,16$ & 0,41 & $-1,56$ & $-16,16$ & $-7,85$ \\
\hline $\mathrm{DE}$ & Alemanha & 25,39 & 25,32 & $-0,27$ & $-0,01$ & 0,01 & $-0,25$ & $-0,03$ \\
\hline JP & Japão & 14,14 & 21,17 & 49,68 & $-1,53$ & 4,30 & 11,19 & 35,72 \\
\hline FR & França & 5,63 & 4,66 & $-17,24$ & 0,25 & $-0,11$ & $-5,40$ & $-11,98$ \\
\hline GB & Reino Unido & 5,02 & 3,25 & $-35,25$ & $-11,43$ & 6,33 & 0,65 & $-30,80$ \\
\hline $\mathrm{KR}$ & Coreia do Sul & 0,28 & 2,27 & 711,81 & 39,95 & 14,96 & 499,79 & 157,11 \\
\hline TW & Taiwan & 0,14 & 0,49 & 261,49 & 286,08 & 343,88 & $-56,01$ & $-312,46$ \\
\hline RU & Rússia & 0,09 & 0,13 & 49,14 & 199,88 & 19,40 & $-38,47$ & $-131,67$ \\
\hline $\mathrm{CN}$ & China & 0,06 & 0,46 & 692,54 & 21,16 & 11,39 & 598,37 & 61,61 \\
\hline BR & Brasil & 0,05 & 0,09 & 88,89 & 91,43 & $-8,52$ & $-10,29$ & 16,27 \\
\hline
\end{tabular}

Fonte: EPO (2012). Elaboração do autor.

No Brasil, um maior desempenho dos seus depositantes residentes resultou no aumento absoluto da quantidade de patentes e no crescimento de $88,89 \%$ da sua participação entre os períodos. Essa ampliação pode ser explicada, principalmente, pelo maior dinamismo da sua atividade patenteadora, verificado pelo efeito technology share. Para composição desse efeito, foram fundamentais os resultados alcançados nos setores energias alternativas e agricultura.

Entretanto, o país deixou de aumentar ainda mais sua participação por não registrar um correto padrão de especialização no período inicial, dado seu efeito structural technology ter sido negativo. Contribuiu para esse desempenho a forte participação do setor gerenciamento de resíduo que perdeu participação global entre os períodos analisados. A especialização "incorreta” revelada pelo efeito structural technology é uma característica particular do Brasil, se considerarmos o conjunto 
dos países fast followers, e tem importantes impactos restritivos no seu dinamismo tecnológico. Se, por um lado, a "correta" especialização significa a atuação em áreas dinâmicas e com alto nível de oportunidade, por outro, a especialização em áreas estagnadas tende a limitar o movimento em direção aos campos mais ativos e pode exigir a ação coordenada de políticas públicas no sentido de uma nova trajetória tecnológica (JUNGMITTAG, 2004).

Outra explicação para esse resultado é dada pelo efeito technology adaptation, que foi positivo e significa que o abandono de áreas com baixo crescimento no setor gerenciamento de resíduos foi suficiente para compensar o ingresso em áreas estagnadas nos setores transporte e conservação de energia.

\section{Considerações finais}

Este artigo teve como objetivo investigar as transformações na estrutura tecnológica brasileira em áreas com potencial para mitigação das mudanças climáticas entre os períodos 1987-1998 e 1999-2010. A partir de um conjunto de dados originais com patentes em 39 áreas relacionadas a essas tecnologias, o artigo explorou as transformaçōes tecnológicas brasileiras no conjunto das áreas que são percebidas como estratégicas na mitigação de emissões de GEE.

Ainda que países economicamente grandes espalhem sua atividade inovativa numa ampla gama de campos tecnológicos (MANCUSI, 2001, 2003), a especialização brasileira em tecnologias de mitigação das mudanças climáticas concentra-se em aproximadamente um terço do total de áreas e se mostra aquém do conjunto potencial de tecnologias existentes. Esses resultados são um importante indicativo da tendência futura da estrutura tecnológica brasileira, tendo em vista que o caráter cumulativo desse processo condiciona os padrões de especialização no futuro às realizações tecnológicas do passado.

Os resultados alcançados foram sistematizados em duas partes principais, de acordo com os métodos empregados. A primeira refere-se às transformações na especialização tecnológica do país no período pós-Kyoto, que reduziram de 15 para 11 o número de áreas em que o país era especializado, com ingresso em duas áreas e abandono de outras seis. A principal explicação para este resultado é a dificuldade do país em acompanhar a dinâmica internacional de patenteamento nessas áreas. Tais resultados mostram que a persistência tem prevalecido sobre a mobilidade em um nível próximo ao encontrado no estudo de Urraca-Ruiz (2008), com níveis setoriais mais agregados. Também percebe-se que, na maioria dos setores, a variação 
na quota de participação está positivamente relacionada com a vantagem tecnológica obtida no período final, o que, de acordo com Archibugi e Pianta (1992), indica que a trajetória da atividade inovadora tem determinado a especialização tecnológica.

A segunda parte abordou a interpretação das transformações do Brasil em uma perspectiva internacional. Se considerarmos os depósitos de patentes realizados por residentes do Brasil no EPO, percebe-se que o país aumentou em 88,89\% sua participação. A aplicação do método de decomposição estrutural revelou que essa ampliação deveu-se, principalmente, ao maior dinamismo da sua atividade patenteadora, verificado pelo efeito technology share. Por outro lado, um efeito structural technology negativo mostrou que o país teve seu crescimento limitado por não dispor de um correto padrão de especialização no período inicial. Da mesma forma, a explicação dada pelo efeito positivo do technology adaptation mostrou que o movimento de ingresso em áreas com baixo crescimento foi mais do que compensado pela saída de áreas igualmente estagnadas.

Os resultados alcançados buscaram explorar o potencial da metodologia proposta e constituem evidências importantes para orientar futuros trabalhos que busquem novas explicações para as transformações na estrutura tecnológica brasileira nessas áreas. Pesquisas nesse tema devem ser orientadas para uma caracterização mais ampla do perfil dos depositantes de patentes e para uma análise com maior desagregação setorial.

\section{Referências bibliográficas}

ALBUQUERQUE, E. M. Domestic patents and developing countries: arguments for their study and data from Brazil (1980-1995). Research Policy, v. 29, n. 9, p. 1.047-1.060, 2000. ANTONELLI, C.; CRESPI, F.; SCELLATO, G. Inside innovation persistence: new evidence from Italian micro-data. Structural Change and Economic Dynamics, v. 23, p. 341-353, 2012. ARCHIBUGI, D.; PIANTA, M. Specialisation and size of technological activities in industrial countries: the analysis of patent data. Research Policy, v. 21, p. 79-93, 1992.

Aggregate convergence and sectoral specialization in innovation. Journal of Evolutionary Economics, v. 4, p. 17-33, 1994.

ARCHIBUGI, D.; FILIPPETTI, A.; FRENZ, M. Economic crisis and innovation: is destruction prevailing over accumulation? Research Policy, v. 42, p. 303-314, 2013.

ARROW, K.; COHEN, L.; DAVID, P.; HAHN, R.; ROBERT, W.; KOLSTAD, C.; LANE, L.; MONTGOMERY, D.; NELSON, R. R.; NOLL, R.; SMITH, A. E. A statement 
on the appropriate role for research and development in climate policy. The Economist's Voice, v. 6, n. 1, p. 1-4, 2009.

BALASSA, B. Trade liberalisation and revealed comparative advantage. The Manchester School of Economic and Social Studies, n. 33, 1965.

CANTWELL, J.; ANDERSEN, B. A statistical analysis of corporate technological leadership historically. Economics of Innovation and New Technology, v. 4, p. 211-234, 1996.

CEFIS, E.; ORSENIGO, L. The persistence of innovative activities a cross-countries and cross-sectors comparative analysis. Research Policy, v. 30, p. 1139-1158, 2001.

CEFIS, H. Is there persistence in innovative activities? International Journal of Industrial Organization, v. 21, p. 489-515, 2003.

COHEN, W. M.; NELSON, R. R.; WALSH, J. P. Protecting their intellectual assets: appropriability conditions and why U.S. manufacturing firms patent (or not). Cambridge, MA: National Bureau of Economic Research - NBER, 2000 (Working paper, n. 7552).

DECHEZLEPRÊTRE, A. Fast-tracking "green" patent applications: an empirical analysis. Grantham Research Institute on Climate Change and the Environment, 2013 (Working paper, 107).

DECHEZLEPRÊTRE, A.; GLACHANT, M.; HASCIC, I.; JOHNSTONE, N.; MÉNIÈRE, Y. Invention and transfer of climate change mitigation technologies: a global analysis. Review of Environmental Economics and Policy, v. 5, n. 1, p. 109-130, 2011.

DERNIS, H.; GUELLEC, D.; POTTERIE, B. V. P. Using patent counts for cross-country comparisons of technology output. The STI Review, v. 27, p. 129-46, 2001.

EPO - European Patent Office. EPO Worldwide Patent Statistical Database (EPO PATSTAT). Viena: EPO, 2012.

FABER, A.; FRENKEN, K. Models in evolutionary economics and environmental policy: towards an evolutionary environmental economics. Technological Forecasting and Social Change, v. 76, n. 4, p. 462-470, 2009.

FAGERBERG, J.; SOLLIE, G. The method of constant-market-shares analysis reconsidered. Applied Economics, v. 19, p. 1571-1585, 1987.

GRILICHES, Z. Patent statistics as economic indicators: a survey. Journal of Economic Literature, v. 28, n. 4, p. 1661-1707, 1990.

GRUPP, H.; SCHOMACH, U. Patent statistics in the age of globalization: new legal procedures, new analytical methods, new economic interpretation. Research Policy, v. 28, p. 377-396, 1999. 
HORBACH, J.; RAMMER, C.; RENNINGS, K. Determinants of eco-innovations by type of environmental impact - The role of regulatory push/pull, technology push and market pull. Ecological Economics, v. 78, p. 112-122, 2012.

HUANG, H.; MIOZZO, M. Patterns of technological specialisation in Latin American and East Asian countries: an analysis of patents and trade flows. Economics of Innovation and New Technology, v. 13, n. 7, p. 615-653, 2003.

INPI - Instituto Nacional da Propriedade Industrial. Base de Dados sobre Propriedade Intelectual para fins Estatísticos (BADEPI). Rio de Janeiro: Inpi, 2013.

JUNGMITTAG, A. Innovations, technological specialisation and economic growth in the EU. International Economics and Economic Policy, v. 1, p. 247-273, 2004.

KEMP, R.; PEARSON, P. Final report MEI project about measuring eco-innovation. Maastricht, 2008. Disponível em: <https://www1.oecd.org/env/consumption-innovation/43960830. pdf >. Acesso em: 15 ago. 2014.

KLEVORICK, A. K.; LEVIN, R. C.; NELSON, R. R.; WINTER, S. G. On the sources and significance of inter-industry differences in technological opportunities. Research Policy, v. 24, p. $185-205,1995$.

LAURSEN, K. The impact of technological opportunity on the dynamics of trade performance. Structural Change and Economic Dynamics, v. 10, p. 341-357, 1999.

LUCCHESI, A. Environmental innovations: evidence from Brazilian manufacturing firms. 170f. Tese (Doutorado em Economia do Desenvolvimento) - Universidade de São Paulo, São Paulo, 2013.

MALERBA, F; ORSENIGO, L. Schumpeterian patterns of innovation are technology-specific. Research Policy, v. 25, p. 451-478, 1996.

MALERBA, F.; ORSENIGO, L.; PERETTO, P. Persistence of innovative activities, sectoral patterns of innovation and international technological specialization. International Journal of Industrial Organization, v. 15, p. 801-826, 1997.

MALERBA, F.; MONTOBBIO, F. Exploring factors affecting international technological specialization. Journal of Evolutionary Economics, v. 13, p. 411-434, 2003.

MANCUSI, M. L. International technological specialization in industrial countries: patterns and dynamics. Weltwirtschaftliches Archiv, v. 137, n. 4, p. 593-621, 2001. - Geographical concentration and the dynamics of countries' specialization in technologies. Economics of Innovation and New Technology, v. 12, n. 3, p. 269-291, 2003. 
MELICIANI, V. The impact of technological specialisation on national performance in a balance-of-payments-constrained growth model. Structural Change and Economic Dynamics, v. 13, p. 101-118, 2002.

MONTOBBIO, F.; RAMPA, F. The impact of technology and structural change on export performance in nine developing countries. World Development, v. 33, n. 4, p. 527-547, 2005. MOWERY, D. C.; NELSON, R. R.; MARTIN, B. R. Technology policy and global warming: why new policy models are needed (or why putting new wine in old bottles won't work). Research Policy, v. 39, n. 8, p. 1011-1023, 2010.

NELSON, R. R.; WOLFF, E. N. Factors behind cross-industry differences in technical progress. Structural Change and Economic Dynamics, v. 8, p. 205-220, 1997.

PICCI, L.; SAVORELLI, L. The technological specialization of countries: an analysis of patent data. St Andrews, Escócia: Universidade de St Andrews, 2013 (School of Economics and Finance Discussion Paper, n. 1301).

PORTO, G.; KANNEBLEY, S.; BARONI, J.; ROMANO, A. Rotas tecnológicas e sistemas de inovação (relatório final). Economia de baixo carbono: avaliação de impactos de restrições e perspectivas tecnológicas. Ribeirão Preto: USP, 2012. Disponível em: <http://www.ebc. fearp.usp.br/arq_docs/Rotas_012013.pdf>. Acesso em: 29 maio 2014.

PUJARI, D. Eco-innovation and new product development: understanding the influences on market performance. Technovation, v. 26, p. 76-85, 2006.

QUEIROZ, J. M.; PODCAMENI, M. V. B. Estratégia inovativa das firmas brasileiras: convergência ou divergência com as questôes ambientais? Revista Brasileira de Inovação, Campinas, v. 13, n. 1, p. 187-224, 2014.

RENNINGS, K. Redefining innovation: eco-innovation research and the contribution from ecological economics. Ecological Economics, v. 32, p. 319-332, 2000.

SOETE, L. The impact of technological innovation on international trade patterns: the evidence reconsidered. Research Policy, v. 16, n. 2-4), p. 101-130, 1987.

STERN, N. The economics of climate change: the stern review. Cambridge: Cambridge University Press, 2007.

UCHIDA, Y.; COOK, P. The transformation of competitive advantage in East Asia: an analysis of technological and trade specialization. World Development, v. 33, n. 5, p. 701-728, 2005.

URRACA-RUIZ, A. Persistência versus mudança estrutural da especialização tecnológica do Brasil. Economia e Sociedade, Campinas, v. 17, n. 3 (34), p. 403-427, 2008. 
. The 'technological' dimension of structural change under market integration. Structural Change and Economic Dynamics, v. 27, p. 1-18, 2013.

URRACA-RUIZ, A.; DURÁN-ROMERO, G. World competences capture by multinationals in environmental technologies. Transnational Corporations Review, v. 5, n. 4, p. 37-53, 2013. VAN ZEEBROECK, V.; POTTERIE, B. V. P.; HAN, W. Issues in measuring the degree of technological specialisation with patent data. Scientometrics, v. 66, n. 3, p. 481-492, 2006. 